\title{
ESTIMATION OF BILIRUBIN IN SERUM
}

\author{
BY \\ W. G. DANGERFIELD AND R. FINLAYSON \\ From the Department of Pathology, St. Bartholomew's Hospital, London
}

(RECEIVED FOR PUBLICATION APRIL 10, 1953)

Most methods of estimating the concentration of bilirubin in serum are based on the van den Bergh reaction in which bilirubin couples with diazotized sulphanilic acid to give red "azobilirubin," the reaction being facilitated by the addition of an activator or catalyst such as ethanol. The many modifications of this reaction can be classified into two groups: (a) those in which ethanol is used as the catalyst, with consequent precipitation of the serum proteins, and $(b)$ those in which some substance, other than ethanol, acts as the catalyst and protein precipitation is avoided. Precipitation methods, such as those described by McNee and Keefer (1925), Haslewood and King (1937), and King and Coxon (1950), suffer from the disadvantage that some " azobilirubin " is lost on the protein precipitate ; and further, the volume of coloured supernatant fluid or filtrate is unknown and varies from test to test. Consequently such methods cannot give really accurate results.

Non-precipitation methods include those published by Jendrassik and Cleghorn (1936) modified by Jendrassik and Grof (1938), Malloy and Evelyn (1937), Rappaport and Eichhorn (1943), Powell (1944), and Patterson, Swale, and Maggs (1952). The technique of Jendrassik and Cleghorn has been criticized by a number of workers on account of clouding of the final test solution. In Malloy and Evelyn's method the colour of the test solution, except in cases of moderate or severe jaundice, is too pale for accurate matching with the standard. The technique of Rappaport and Eichhorn is unsatisfactory for two reasons: the colour of the test solution is unstable and secondly, with some sera, full colour development of the "azobilirubin" compound is delayed for several hours. In Powell's method, serum, treated with diazo and sodium benzoate urea solutions, is matched against an alcohol-activated azobilirubin standard; the use of a standard differing so greatly in composition from that of the test is very undesirable. We have no experience of the method of Patterson, Swale, and Maggs which was published when the present work was almost complete, but we feel that it may be criticized on the ground that the use in the routine laboratory of such a corrosive reagent as $90 \%$ phenol is undesirable.

In the method which we have developed the serum is treated with diazo reagent and a mixture of caffeine sodium benzoate and a phosphate buffer ; the azobilirubin is formed rapidly and it is measured in a photo-electric colorimeter and compared with that of an azobilirubin standard previously prepared from bilirubin dissolved in $\mathrm{N} / 10$ sodium carbonate solution. A blank is used to compensate for any slight cloudiness or any colour other than bilirubin, which may be present in the test serum. This method, which is based on Goodson and Sheard's (1940) technique for estimating urinary bilirubin, is rapid, simple to perform, and gives good colours, the final test and standard solutions being buffered to a $p \mathrm{H}$ of between 5.7 and 6.3. Estimations of serum bilirubin levels by this technique compare well with estimations by the protein-precipitation method of King and Coxon. We have also made some observations on the factors required to bring about satisfactory coupling of bilirubin and diazo solution.

\section{Experimental}

Reagents. -The following reagents are required : Diazo Reagent

Diazo A.-Sulphanilic acid ... ... 1 g.

Concentrated hydrochloric $\begin{array}{llllll}\text { acid } & \ldots & \ldots & \ldots & 15 \mathrm{ml} \text {. }\end{array}$ Distilled water to $\quad \ldots \quad 1,000 \mathrm{ml}$. $\begin{array}{cccc}\text { Diazo B.- } & \text { Sodium nitrite } \ldots & \ldots & 0.5 \mathrm{~g} . \\ \text { Distilled water to ... } & \ldots & 100 \mathrm{ml} .\end{array}$

For use $10 \mathrm{ml}$. of diazo A is mixed with $0.3 \mathrm{ml}$. of diazo B.

Diazo Blank

Concentrated hydrochloric acid $\ldots 15 \mathrm{ml}$.

Distilled water to $\quad \ldots \quad \ldots \quad \ldots \quad 1,000 \mathrm{ml}$.

Caffeine Buffer Mixture.-Caffeine, $25 \mathrm{~g}$., and $25 \mathrm{~g}$. sodium benzoate are dissolved in about $400 \mathrm{ml}$. warm distilled water, cooled, and $4.1 \mathrm{~g}$. potassium dihydro- 
gen phosphate and $3.4 \mathrm{ml}$. $\mathrm{N}$ sodium hydroxide are added. The mixture is diluted to $500 \mathrm{ml}$. with water. The mixture, which should be filtered if not clear, will keep for at least a month.

Bilirubin Solution.-About $20 \mathrm{mg}$. of pure bilirubin (Hoffman la Roche) is weighed accurately and $0.2 \mathrm{ml}$. wetting agent added $(10 \%$ "lissapol" $\mathrm{N}$ or $10 \%$ "teepol"). When wetting is complete $7 \mathrm{ml}$. of $\mathrm{N} / 5$ sodium hydroxide is added and the mixture is stirred until the bilirubin is completely dissolved. It is diluted with $\mathrm{N} / 10$ sodium carbonate to a volume of $500 \mathrm{ml}$. It is important that this preparation should be carried out rapidly (within five minutes). Immediately after this solution has been made it should be used for the preparation of the standard azobilirubin and standard blank solution as below.

Azobilirubin Standard.-This standard solution should be prepared in duplicate. To $50 \mathrm{ml}$. of caffeine buffer mixture $5 \mathrm{ml}$. of diazo solution is added, followed by $10 \mathrm{ml}$. of bilirubin solution, mixed, and allowed to stand for 10 minutes. Then its optical density (S) is measured using a green filter. If stored in the dark this solution can be used for checking the photometer in the subsequent serum determinations made during the following two weeks.

Standard Blank Solution.-This solution also should be prepared in duplicate. To $10 \times 1$. of caffeine buffer mixture $1 \mathrm{ml}$. water is added, followed by $2 \mathrm{ml}$. of bilirubin solution. The solution is mixed and allowed to stand for 10 minutes, then its optical density (SB) is measured using the same green filter as employed in measuring the density of the standard.

\section{Method}

Test and Blank.-Fresh serum, $1 \mathrm{ml}$, is pipetted into each of two test-tubes. To one tube (the test) $0.5 \mathrm{ml}$. diazo reagent is added and to the other tube (the blank) $0.5 \mathrm{ml}$. diazo blank. The tubes are shaken and stood for approximately one minute and it is noted if a red develops in the test solution indicating a positive direct van den Bergh reaction. To both tubes $5 \mathrm{ml}$. of caffeine-buffer mixture is added. The contents are mixed well and stood for 15 minutes to allow for full colour development. The colour is stable for at least one hour.

The optical densities of the test (T) and the blank (B) are measured in a photoelectric colorimeter using a green light filter (e.g., Ilford bright spectrum green 624 , or spectrum green 604 , or, less good, Ilford tricolour green 404) or in a spectrophotometer at $525 \mathrm{~m} \mu$, making the zero setting with water.

Calculation.-Concentration of bilirubin in serum $=\frac{T-B}{S-S B} \times \frac{W}{5}$ in $\mathrm{mg}$. per $100 \mathrm{ml}$. serum where $\mathrm{W}=$ weight of bilirubin in $\mathrm{mg}$. taken in preparing the bilirubin solution, and $T, B, S$, and $S B$, the optical densities of the solutions indicated above. If exactly $20 \mathrm{mg}$. of bilirubin was weighed out the standard azobilirubin solution is equivalent to a serum bilirubin concentration of $4 \mathrm{mg} . / 100 \mathrm{ml}$.

With strongly jaundiced sera (above $6 \mathrm{mg}$. per $100 \mathrm{ml}$.) the test solution is diluted without delay after coupling with a mixture containing $10 \mathrm{vol}$. caffeine-buffer solution and 1 vol. diazo reagent. This diluted solution is allowed to stand for 15 minutes before measuring its optical density.

If only $1 \mathrm{ml}$. (or less) of serum is available sufficient caffeine-buffer mixture is added to make a total volume of $6 \mathrm{ml}$., the optical density of this mixture is measured, and then $0.5 \mathrm{ml}$. diazo reagent is added and the density measured again. This method suffers from the disadvantage that it gives no measure of the direct van den Bergh reaction.

Three or four preparations of standard azobilirubin should be made from the solid bilirubin, and the average value of the factor $\frac{1}{\mathrm{~S}-\mathrm{SB}} \times \frac{\mathrm{W}}{5}$ determined; this value is then used for calculating all serum deterninations.

In order to check the photometer some stable standard red solution should be measured each time a saru.n bilirubin level is determined. The azobilirubin standard can be used for this purpose, but its density very gradually increases. An alternative and more permanent standard which may be used is the methyl red solution prepared according to the method of King and Coxon (1950), although the colour density of this solution is much less than that of the azobilirubin standard and its tint less blue than the latter.

\section{Results}

After this method had been chosen it was necessary to determine (1) the purity of samples of bilirubin commercially available, and (2) to investigate $\frac{}{0}$ the stability of the standard solutions of bilirubin $\bar{\gamma}$ and azobilirubin. As regards the purity of bilirubin, two preparations of bilirubin were examined $\frac{\partial}{3}$ by chromatographic adsorption of the chloroform solution on alumina and eluting with chloroform 을 containing $1 \%$ acetic acid. It was found that the preparation made by Hoffman la Roche was very nearly pure and this was chosen as the standard.

As regards the stability of the bilirubin and or azobilirubin solutions, it was found that standard $\mathrm{O}$ bilirubin solutions prepared in the manner $\mathbb{\omega}$ described above showed no alteration in concen- $\widetilde{\gamma}$ tration when examined five, 10 , and 15 minutes after preparation. While we did not care to use $\frac{\bar{\Phi}}{\mathbb{Q}}$ bilirubin solutions more than 10 minutes after $\stackrel{\infty}{+}$ preparation, we observed that on some occasions $\underset{T}{-}$ they remained stable for at least one hour, but at $\bar{P}$ other times the standard was found to have lost $\stackrel{\mathbb{Q}}{\stackrel{Q}{Q}}$ $5 \%$ of its bilirubin half an hour after preparation. $\stackrel{\varnothing}{\varrho}$

In contrast the standard solutions of azobilirubin were found to be much more stable. After adding the bilirubin to the mixed caffeine buffer and diazo 


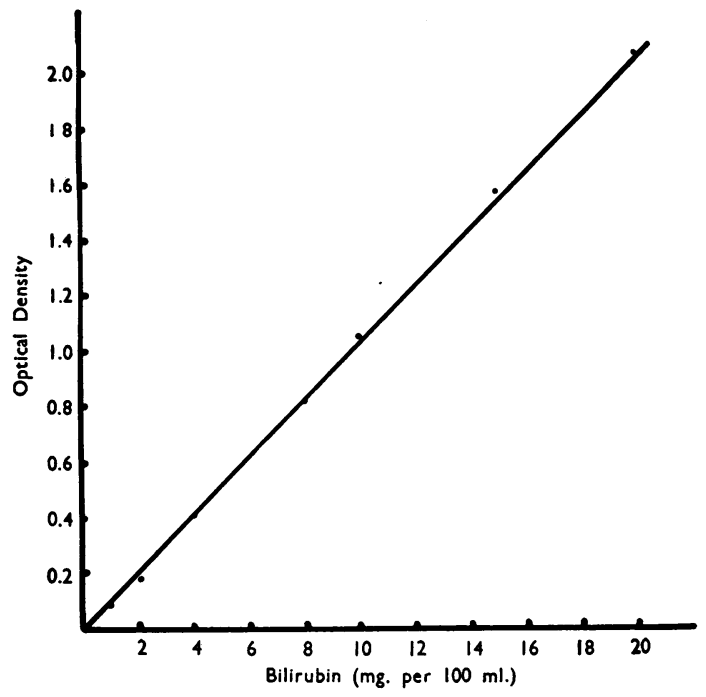

FIG. 1.-Relationship between the concentration of bilirubin ( $\mathrm{mg}$. per $100 \mathrm{ml}$.) and the optical density of azobilirubin formed.

solutions, the colour developed rapidly and reached its full intensity within a minute. Occasionally a very small decrease in optical density occurred during the next few minutes, but from 10 minutes onwards the extinction remained constant for two weeks, and thereafter very slowly increased.

\section{Relationship between Bilirubin Concentration and} Optical Density of Azobilirubin.-A graph (Fig. 1) was made by plotting the optical density of azobilirubin solutions, prepared from a series of dilutions of a $20 \mathrm{mg}$. per $100 \mathrm{ml}$. bilirubin standard against the concentration of bilirubin expressed in mgm. per $100 \mathrm{ml}$. The graph was practically a straight line between $2 \mathrm{mg}$. and $20 \mathrm{mg}$. bilirubin per $100 \mathrm{ml}$. Further experiments showed that even up to concentrations of $40 \mathrm{mg}$. per 100 $\mathrm{ml}$. the departure from the straight line was only slight.

Recovery Experiments.-The addition of alkaline solutions of bilirubin in concentrations ranging from 2 to $8 \mathrm{mg}$. per $100 \mathrm{ml}$. to both normal and jaundiced sera gave " recoveries" of $95 \%$ to $103 \%$.

Comparison with King and Coxon (1950) Method.-Parallel serum bilirubin estimations were made by the proposed caffeine method and the modification of the Haslewood-King technique described by King and Coxon (1950). The results from 32 consecutive sera obtained from nonjaundiced patients are shown in Table $I$. The results from 42 cases of hyperbilirubinaemia are shown in Table II.
TABLE I

BILIRUBIN CONCENTRATION OF 32 CONSECUTIVE SERA FROM NON-JAUNDICED PATIENTS

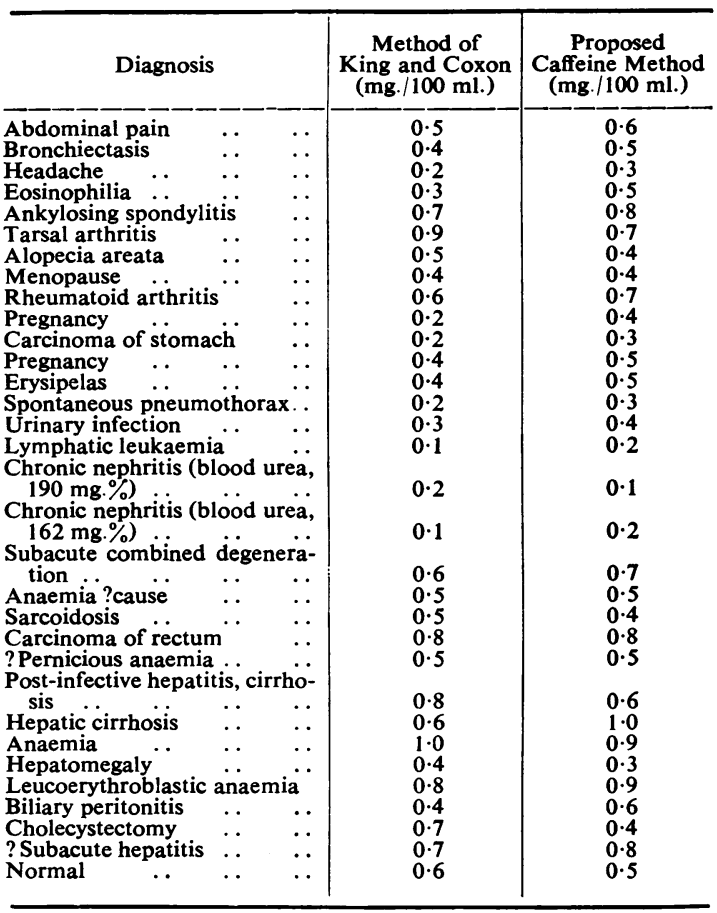

\section{Discussion}

During our search for a good bilirubin standard we carried out many preliminary experiments with a view to determining the conditions governing satisfactory coupling of bilirubin with diazotized sulphanilic acid.

It was found desirable to have a buffer present in order to regulate the rate of coupling. The reaction proceeds only slowly in strongly acid solution, but is very rapid in neutral or alkaline solution. Further, the colour of the azobilirubin formed varies with reaction, being violet in the presence of mineral acid, the usual red or pink at

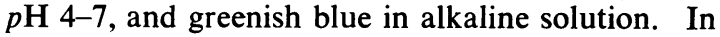
the case of the ordinary van den Bergh test the serum itself is a sufficient buffer to reduce the acidity of the diazo solution to the useful $p \mathrm{H}$ range.

The stability of the azobilirubin formed is dependent on the reaction of the medium (it decomposes rapidly in alkaline solution) and on the presence of other substances, e.g., caffeine, in the solution. In fact caffeine appears both to facilitate coupling and to stabilize the product. 
TABLE II

BILIRUBIN CONCENTRATION OF FORTY-TWO HYPERBILIRUBINAEMIC SERA

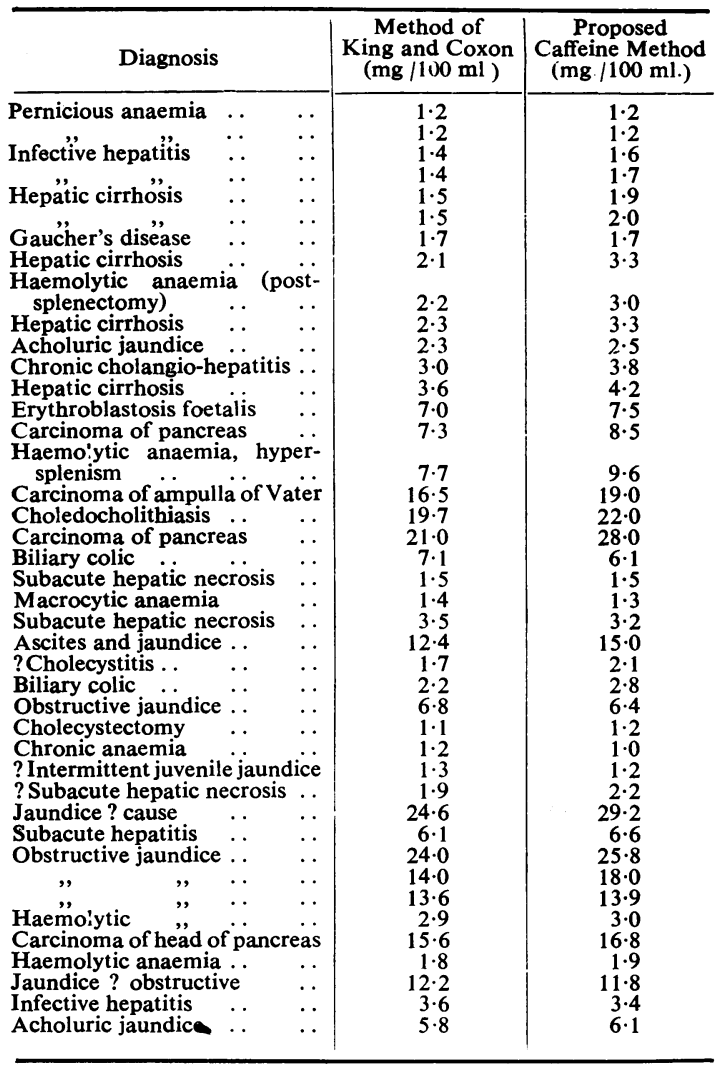

It is desirable that any method of estimation of bilirubin should be capable of being standardized against pure bilirubin and, further, the substances and conditions used for effecting the reaction in the case of the test serum and in the case of the standard should be, as nearly as possible, the same. Comparison of the hue and intensity of a solution of azobilirubin obtained using an alcohol catalyst with one of equal strength obtained by the present method showed that the latter had a slightly bluer tint than the former, and its optical density, using a green light filter, was $4 \%$ less than the former. It is therefore undesirable to use caffeine as a catalyst for the test serum and alcohol for the bilirubin standard.

The only common solvent in which bilirubin is freely soluble and gives a stable solution is chloroform. Consequently a chloroform solution has been used by most workers as the starting point for preparing azobilirubin standards. However, since the coupling reaction takes place in aqueous or aqueous alcoholic solution, it is necessary to find a means of getting the bilirubin from thes chloroform into a water miscible solution. Therछ are two simple methods that can be used for this purpose: (1) dilution of the chloroform solutions with a considerable quantity of alcohol to give ang "alcohol-chloroform solution of bilirubin," andw (2) evaporation of a small quantity of chloroform solution and redissolving the bilirubin in dilute alkali, e.g., sodium carbonate solution. Experi $\vec{\omega}$ ments were carried out with these two solutionso both of which are unstable, particularly the alka용 line one, to see if either is suitable as a standarbs for bilirubin determination. It was found that although the alcohol diluted solution is a suitable $\vec{\omega}$ standard for methods in which alcohol is used as of catalyst, it is not satisfactory for methods employ? ing caffeine as catalyst, and, in fact, an alkaline $\overrightarrow{ }$ solution seemed essential for the present technique The method of evaporating a small quantity of chloroform solution to dryness and redissolving it in alkali, tried initially, was not satisfactory be cause a small loss of bilirubin occurred during $\omega$ evaporation. Consequently the chloroform solu믕 tion was abandoned, and solid bilirubin weighed out each time a standard was made. After con $\bar{\partial}$ siderable experiment a satisfactory method of preparing the bilirubin and azobilirubin solutions was devised.

In order to obtain complete and rapid couplings it is necessary to ensure that the bilirubin stays in solution after it has been added to the reaction. mixture. If any bilirubin is allowed to precipitate out before the diazo solution is added it will no? react and coupling is incomplete. In order to minimize the chance of bilirubin separating out during the preparation of the azobilirubin standaro for our proposed technique the reagents werह mixed in the following manner.. The diazo solus tion was added to the caffeine buffer solution, and then the bilirubin solution was added to the mix ture.

It would be much too laborious and too expenn sive to prepare an azobilirubin standard each tim\& a determination is made. Fortunately it was foung that the standard gives a nearly constant reading and the calibration curve (Fig. 1) is for practicat purposes a straight line passing through the origines Therefore it is sufficiently accurate to calculate the concentration by simple ratio as stated above Using such a method it is important to check th photometer with some stable red solution ever\% time a determination is made. The azobilirubif solution keeps for about two weeks but thereafter may very slowly darken. The methyl red standarg 
of King and Coxon is more stable and.was used as an artificial standard in spite of the fact that it is weaker and less blue in tint than the $4 \mathrm{mg}$. azobilirubin standard. Another solution that may be used as an artificial standard is a $4 \%$ anhydrous cobalt sulphate solution to which an equal volume of $8 \mathrm{~N}$ hydrochloric acid has been added (White, 1932).

For accurate determination it is necessary to compensate for the optical density of the bilirubin which is present in the serum blank (B) but absent from the coupled test solution $(T)$. Consequently $\mathbf{T}-\mathbf{B}$ is not a true measure of the azobilirubin in the test solution but is, in fact, too low, and if the expression $\frac{T-B}{S}$ were used in calculating, the result would also be too low (by about $8 \%$ ). The extent of the correction to be applied is determined by preparing a solution containing the same concentration of bilirubin as used in making the standard azobilirubin solution, but water is substituted for diazo reagent. The optical density of this yellow standard blank solution (SB) is measured with the green light filter. It is found that SB is about $8 \%$ of $S$. The correction for the bilirubin present in the serum blank (B) but not in the test (T) can be made by subtracting SB from $S$ and thus using the expression $\frac{T-B}{S-S B}$ in calculating the bilirubin content.

The method which we have described is suitable for routine laboratory use as it is rapid and simple to perform, and no unusual or corrosive reagents are required. The method can be carried out satisfactorily on slightly haemolysed specimens of serum. Another advantage of the technique is that with strongly jaundiced sera the extra dilution is made after the addition of the diazo solution. In some methods involving protein precipitation it is necessary to dilute the serum with buffer before adding the diazo solution, and considerable experience is required in assessing the degree of pre- liminary dilution necessary to obtain a satisfactory result when using these methods.

We are not aware of any serious disadvantage in the method we present. The most difficult part of the estimation seems to be the preparation of azobilirubin standards of consistent optical density; we found that variations of up to $\pm 3 \%$ from the mean may occur.

The main criticism of caffeine catalysed methods in general is the fact that the final solution sometimes clouds, perhaps due to separation of benzoic acid derived from the catalyst solution. In our method benzoic acid never separated, and the final solutions were nearly always satisfactorily clear. On rare occasions the final solution has a very slight milkiness, possibly due to a globulin or lipoid component in the serum. In the three cases so far encountered the milkiness appeared to be present to an equal extent in the test and blank solutions, and there was no discrepancy between the results obtained by this method and the method of King and Coxon.

\section{Summary}

A simple and rapid method for the estimation of bilirubin in serum is described. The method is suitable for routine use in laboratories using photoelectric colorimeters. A number of observations are made on the conditions necessary for satisfactory reaction between bilirubin solutions and diazotized sulphanilic acid.

We wish to thank Dr. H. E. Archer for his helpful advice in the preparation of this paper, and Miss E. M. Pusey for carrying out some of the determinations.

\section{REFERENCES}

Goodson, W. H., and Sheard. C. (1940). J. Lab. clin. Med., $26,423$. Haslewood, G. A. D., and King, E. J. (1937). Biochem J., 31, 920. Jendrassik, L.. and Cleghorn. R. A. (1936). Biochem. Z., 289, 1. C and Grof, P. (1938). Ibid. 297, 81.

King, E. J., and Coxon, R. V. (1950). Journal of Clinical Pathology, 3,248 .

Malloy, H. T., and Evelyn, K. A. (1937). J. biol. Chem , 119, 481. McNee, J. W., and Keefer, C. S. (1925). Brit. med. J., 2, 52.

Patterson, J., S'wale. J., and Maggs, C. (1952). Biochem. J., 52, 100.

Powell, W. N. (1944). Amer. J. clin. Path, 11, technical section, 8, 55.

Rappaport, F., and Eichhorn, F. (1943). Lancet, 1, 62.

-White, F. D. (1932). Brit. J. exp. Path., 13, 76 